\title{
Sex ratio theory in geographically structured populations
}

\section{G. Bulmer}

Department of Biomathematics, 5 South Parks Road, Oxford OX1 3UB, U.K.

Equilibrium sex ratios have been determined analytically under Wright's island model in order to determine the effect of population structure with limited dispersal. When mating occurs before dispersal, the dispersal rate has little or no effect, and the equilibrium sex ratio remains the same as under complete dispersal (the standard model of local mate competition). With dispersal before mating, there is a bias towards the sex with the higher dispersal rate due to lower competition between sibs of that sex.

\section{INTRODUCTION}

Fisher (1930) showed that a 1:1 sex ratio should be evolutionarily stable as there would otherwise be a frequency-dependent advantage to the rarer sex. Hamilton (1967) pointed out that Fisher's argument depends on the assumption of population-wide random mating, and showed that a female-biased sex ratio was expected in a model in which mating occurred within small local subgroups before population-wide dispersal of mated females. Subsequent work on Hamilton's model has been reviewed by Charnov (1982, Chapter 5) and Harvey (1985). Bulmer and Taylor (1980) considered a model of a geographically-structured population in which males and females disperse different distances round a circle, and concluded that the sex ratio is biased in favour of the sex with the higher variability of dispersal distance because of the reduced competition between siblings of that sex. However, mathematical argument was rather heuristic because of the difficulty of obtaining analytic results under this model of dispersal. I shall here consider the sex ratio under Wright's island model of dispersal which is easier to analyse. Although this model is biologically rather implausible, it seems to behave qualitatively like a two-dimensional model with isolation by distance.

\section{MODELS AND METHODS}

Consider a locus with two alleles, $R$ and $S$, acting in females and determining the sex ratio among her offspring. If females are haploid, the genotypes $R$ and $S$ determine sex ratios $r$ and $s$ respectively; if females are diploid the genotypes $R R$ and $S S$ determine the above sex ratios, while $R S$ females have sex ratio $r, s$ or $\frac{1}{2}(r+s)$ according as $S$ is recessive, dominant or additive. (The three types of gene action will be considered separately.) The world contains a large number of islands, in each of which $n$ mated females breed each year, each female having a large number of offspring.

Two models will be considered, mating before dispersal and mating after dispersal. In mating before dispersal, the offspring in each island mate at random among themselves, each female mating once only, a fraction $d$ of the mated females then disperse at random among all the islands, the rest remaining in their natal island, and the mated females now in each island compete for the $n$ available breeding sites. In mating after dispersal, a fraction $d_{1}$ of the female offspring and $d_{2}$ of the males in each island disperse at random among all the islands, the rest remaining in their natal island; mating then occurs at random in each island, followed by competition for the $n$ available breeding sites as before.

The method used in analysing these models is to determine whether $S$ will spread when it is rare. If there are $k$ types of mated females $(k=4$ for haploids, 6 for haplo-diploids and 9 for diploids), there will be

$$
N=(n+k-1) ! / n !(k-1) !
$$

different types of island (Feller, 1968, Theorem II. 5.2), of which $N-1$ will be rare and one (contain- 
ing only $R$ genes) common. The dynamics of the rare types can be represented by the linearised recurrence relationship

$$
\boldsymbol{x}_{t+1}=\boldsymbol{A}(r, s) \boldsymbol{x}_{t}
$$

where $x_{t}$ and $x_{t+1}$ are column vectors of the frequencies of the $N-1$ rare types in years $t$ and $t+1$, and $\boldsymbol{A}(r, s)$ is a square transition matrix of order $N-1$ which can be calculated for any particular model.

If $\lambda(r, s)$ is the dominant eigenvalue of $\boldsymbol{A}(r, s)$, the allele $S$ will increase or decrease in frequency when rare according as $\lambda(r, s)$ is greater or less than unity. The conditions for $r$ to be a locally stable equilibrium sex ratio are that

$$
\begin{aligned}
& \left.\frac{\partial \lambda(r, s)}{\partial s}\right|_{s=r}=0 \\
& \left.\frac{\partial^{2} \lambda(r, s)}{\partial s^{2}}\right|_{s=r}<0 .
\end{aligned}
$$

Equation (3i) is equivalent to

$$
\left.\frac{\partial}{\partial s} \operatorname{det}(\boldsymbol{A}(r, s)-\boldsymbol{I})\right|_{s=r}=0
$$

(Taylor and Bulmer, 1980). In this paper the equilibrium sex ratio was found by solving (4) numerically, and stability was then checked by evaluating $\lambda(r, s)$ in the neighbourhood of the equilibrium. All equilibria found were stable. However, the equilibrium sex ratio in some cases depends on the mode of gene action of the mutant so that no evolutionarily stable sex ratio exists; this will be discussed further in the next section. This technique is limited to small values of $n$ because of limitations on the size of matrix which can be handled by the computer.
RESULTS

\section{Mating before dispersal}

In this model mating occurs before dispersal in each island, a fraction $d$ of the mated females disperse at random among all the islands, the rest remaining in their natal island, and the mated females then compete for the $n$ breeding sites available on each island. With complete dispersal $(d=1)$ this is the model of local mate competition first proposed by Hamilton (1967), for which the evolutionarily stable sex ratio is known analytically to be $r=(n-1) / 2 n$ for haploids and diploids and $r=(n-1)(2 n-1) /(4 n-1) n$ for haplo-diploids (Hamilton, 1967; 1979; Taylor and Bulmer, 1980).

The numerical results in Table 1 show that there is no effect of partial dispersal $(d<1)$ under haploidy or under diploidy with additive gene action. There is however a small effect of the mode of gene action under diploidy, whose effect can best be illustrated by an example. Suppose that $n=2, d=0.1$ under diploidy, and that the genotypes $R R, R S$ and $S S$ produce sex ratios 0.251 , 0.249 and 0.249 respectively. Then both $R$ and $S$ can invade when rare, and a polymorphism with an intermediate sex ratio will result. However, if the dominance were reversed, $R S$ producing sex ratio 0.251 , both the homozygous states become stable equilibria. There is thus strictly speaking no evolutionarily stable sex ratio, but for practical purposes one would expect to find a population close to the sex ratio predicted under additive gene action, and hence conclude that partial dispersal has no effect on the equilibrium sex ratio. The situation is similar to that found under the haystack model by van Tienderen (1984).

Table 1 Equilibrium sex ratio with mating before dispersal

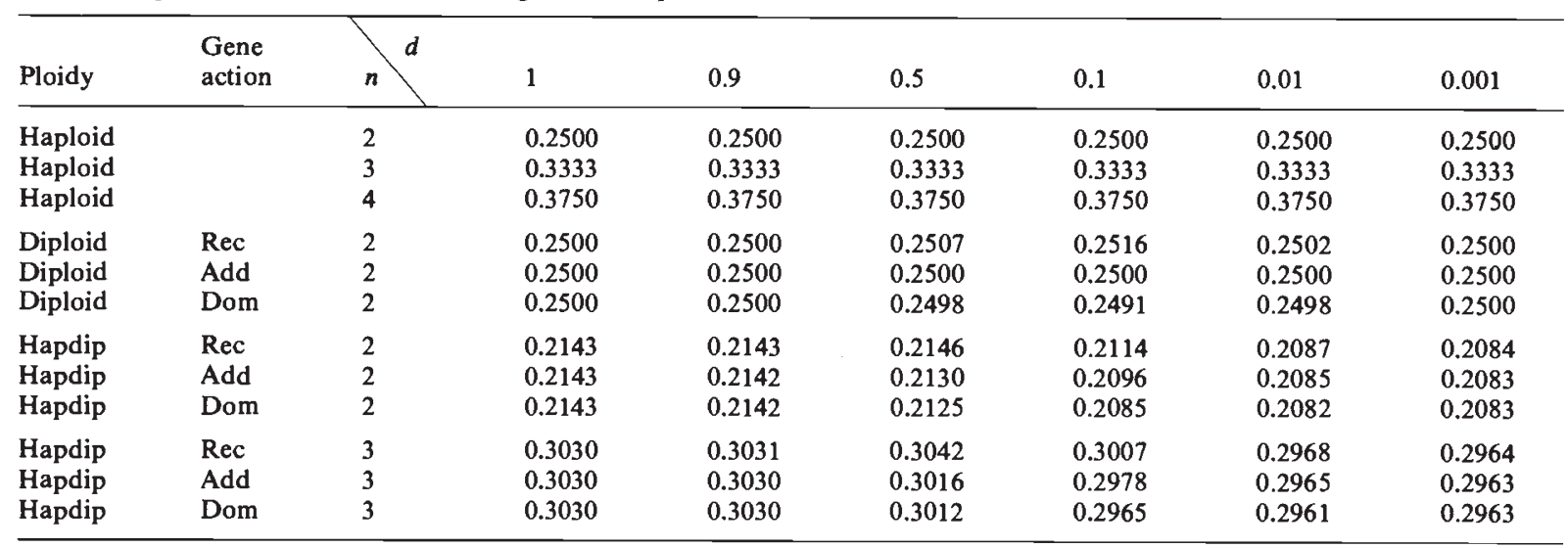


Under haplo-diploidy there is a similar effect of dominance (reading down a column), but there is also a consistent fall in the sex ratio with decreasing dispersal (reading along a row). As $d$ goes from 1 to 0 , the equilibrium sex ratio goes from $3 / 14$ to about $5 / 24$ for $n=2$ and from $10 / 33$ to about $8 / 27$ for $n=3$. An empirical formula which reproduces these values for very low dispersal rates is $r=$ $(n-1)(3 n-1) / 6 n^{2}$.

\section{Mating after dispersal}

In this model a fraction $d_{1}$ of the females and $d_{2}$ of the males in each island disperse at random among all the islands, the rest remaining in their natal island, followed by random mating within each island and competition among the mated females for the $n$ available breeding sites. Table 2 shows the equilibrium sex ratio under haploidy. The main conclusions are that the sex ratio is biased in favour of the sex with the higher dispersal rate (with no bias when $d_{1}=d_{2}$ ), and that the bias decreases as $n$, the local population size increases.
Under diploidy, the equilibrium sex ratio is the same as under haploidy regardless of the mode of gene action for all dispersal rates when $n=1$ and if $d_{1}=d_{2}$ or $d_{1}=1, d_{2}=0$ or $d_{1}=0, d_{2}=1$ when $n=2$. For other dispersal rates there are minor differences which are of no practical importance. For example, with $n=2, d_{1}=0.1, d_{2}=0.5$, the equilibrium sex ratio under diploidy is 0.6598 , 0.6589 or 0.6588 according as the mutant is recessive, additive or dominant, compared with 0.6624 under haploidy.

The equilibrium sex ratio under haplo-diploidy with $n=1$ is shown in table 3 . There are small and rather irregular differences from the values for haploidy and diploidy shown in table $2 \mathrm{a}$.

\section{DISCUSSION}

\section{Mating before dispersal}

For the standard model of local mate competition with complete dispersal $(d=1)$, Taylor (1981)

Table 2 Equilibrium sex ratio with mating after dispersal under haploidy

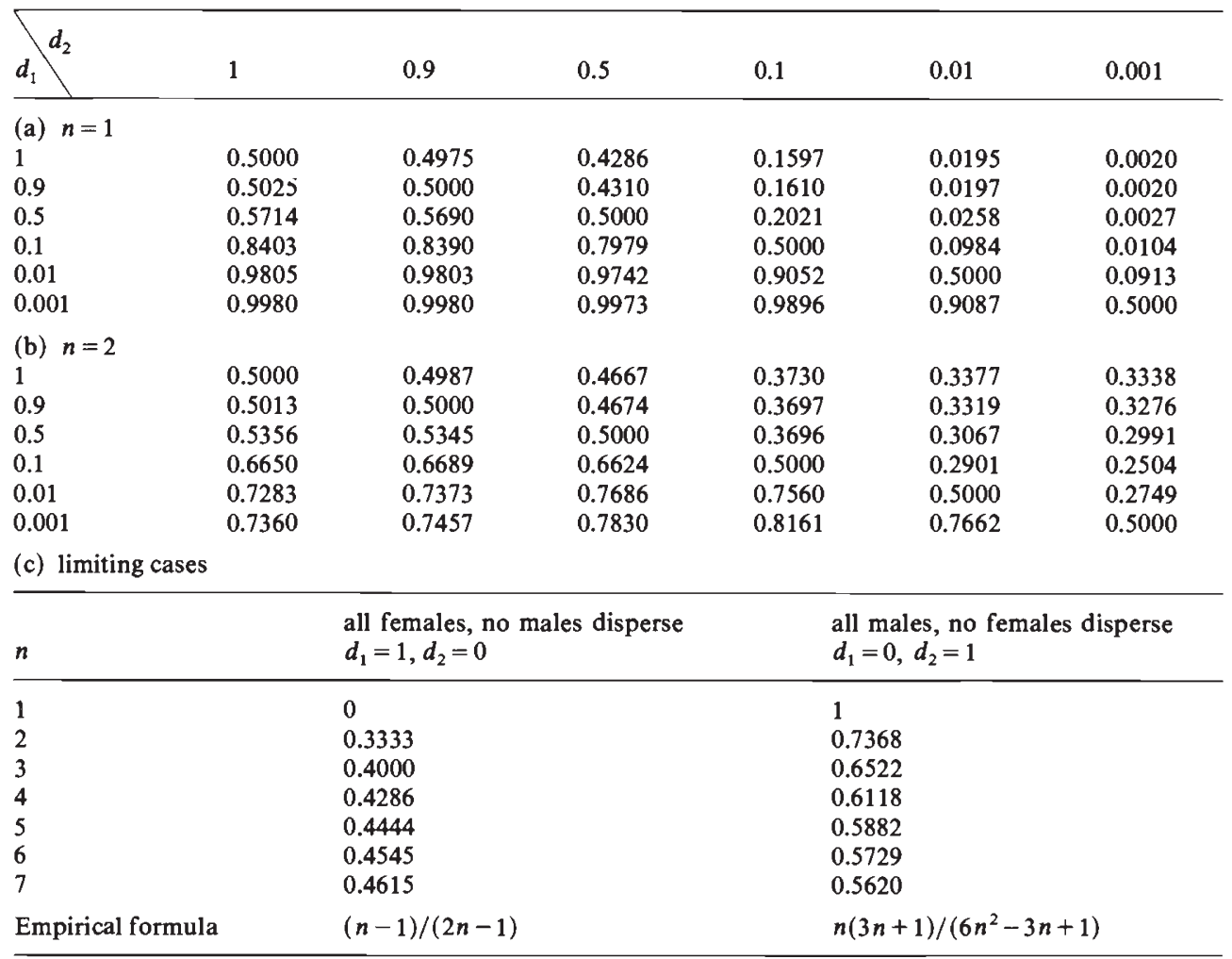


Table 3 Equilibrium sex ratio with mating after dispersal under haplo-diploidy with $n=1$

\begin{tabular}{|c|c|c|c|c|c|c|}
\hline$d_{1}$ & 1 & 0.9 & 0.5 & 0.1 & 0.01 & 0.001 \\
\hline \multicolumn{7}{|c|}{ (a) Recessive gene action } \\
\hline 1 & 0.5000 & 0.4975 & 0.4286 & 0.1597 & 0.0195 & 0.0020 \\
\hline 0.9 & 0.5025 & 0.5012 & 0.4466 & 0.1845 & 0.0238 & 0.0024 \\
\hline 0.5 & 0.5714 & 0.5658 & 0.5226 & 0.2837 & 0.0452 & 0.0048 \\
\hline 0.1 & 0.8403 & 0.8350 & 0.7956 & 0.5375 & 0.1164 & 0.0132 \\
\hline 0.01 & 0.9805 & 0.9797 & 0.9736 & 0.9134 & 0.5360 & 0.1054 \\
\hline 0.001 & 0.9980 & 0.9979 & 0.9973 & 0.9905 & 0.9191 & 0.5357 \\
\hline \multicolumn{7}{|c|}{ (b) Additive gene action } \\
\hline 1 & 0.5000 & 0.4955 & 0.4043 & 0.1346 & 0.0157 & 0.0016 \\
\hline 0.9 & 0.5025 & 0.4991 & 0.4142 & 0.1446 & 0.0172 & 0.0018 \\
\hline 0.5 & 0.5714 & 0.5689 & 0.4938 & 0.2093 & 0.0282 & 0.0029 \\
\hline 0.1 & 0.8403 & 0.8385 & 0.7929 & 0.5167 & 0.1080 & 0.0122 \\
\hline 0.01 & 0.9805 & 0.9802 & 0.9734 & 0.9110 & 0.5334 & 0.1046 \\
\hline 0.001 & 0.9980 & 0.9980 & 0.9973 & 0.9903 & 0.9188 & 0.5355 \\
\hline \multicolumn{7}{|c|}{ (c) Dominant gene action } \\
\hline 1 & 0.5000 & 0.4954 & 0.4000 & 0.1284 & 0.0148 & 0.0015 \\
\hline 0.9 & 0.5025 & 0.4990 & 0.4089 & 0.1358 & 0.0158 & 0.0016 \\
\hline 0.5 & 0.5714 & 0.5691 & 0.4892 & 0.1925 & 0.0246 & 0.0025 \\
\hline 0.1 & 0.8403 & 0.8386 & 0.7922 & 0.5049 & 0.1024 & 0.0115 \\
\hline 0.01 & 0.9805 & 0.9802 & 0.9734 & 0.9092 & 0.5310 & 0.1039 \\
\hline 0.001 & 0.9880 & 0.9980 & 0.9973 & 0.9901 & 0.9185 & 0.5352 \\
\hline
\end{tabular}

identifies two forces leading to a female-biased sex ratio: (i) diminishing returns associated with producing sons because of competition between males for mates, (ii) producing more daughters increases the mating opportunities of sons if sib-mating occurs at random. Grafen $(1984$, p. 81$)$ suggests that a third factor can be added if the females in each group are related. He writes: "Making a daughter would then have the additional benefit of increasing the mating success of sons of relatives. The sex ratio would therefore be even more female biased if the $n$ females were related to each other." One would therefore expect the sex ratio to become more female biased with decreasing dispersal. Indeed, Frank (1985) suggests that the sex ratio in a structured population should be proportional to $P_{S T}$, Wright's index of panmixia, and should therefore decline to zero as the dispersal rate tends to zero.

The main purpose of this study was to investigate this prediction. In fact, table 1 shows clearly that the dispersal rate has little or no effect on the equilibrium sex ratio.

It seems that the kin selection factor of providing daughters to increase the reproductive success of ones relatives' sons is exactly counterbalanced by a fourth factor: making fewer daughters increases the reproductive success of ones relatives' daughters who do not disperse. Stating this in a different way, intra-group altruism does not evolve when density-dependent regulation acts separately within each group, as discussed by Grafen (1984, p. 80).

\section{Mating after dispersal}

Several points emerge from table 2. Firstly, the sex ratio is biased towards the sex with the higher dispersal rate, presumably because of the reduced competition between siblings of that sex. Secondly, ihere is no bias when the dispersal rates are equal $\left(d_{1}=d_{2}\right)$, even when they are very low; this confirms the conclusion of the previous sub-section that population structure with limited dispersal does not in itself lead to a female biased sex ratio. Thirdly, there is a slight asymmetry when $n>1$ in that $r(x, y)+r(y, x)>1$, where $r(x, y)$ is the equilibrium sex ratio with female and male dispersal rates $x$ and $y$. This must be due to a small effect of the assumption that females control the sex ratio; if $r^{*}(x, y)$ is the equilibrium sex ratio under male control, it is clear from symmetry considerations that

$$
r(x, y)+r^{*}(y, x)=1 .
$$

Fourthly, for fixed dispersal rates the bias in the sex ratio decreases as the population size within each island $(n)$ increases because competition between siblings decreases with increasing $n$.

The last point can be understood from the special cases in table $2 \mathrm{c}$. When all females but no males disperse, the equilibrium sex ratio is 
$(n-1) /(2 n-1)$. This result was obtained by Werren (1983) for this model by a heuristic argument, and was derived by Maynard Smith (1985) for a similar model used to study the effect of the first force involved in local mate competition (diminishing returns for producing sons) in the absence of the second force (producing more daughters enhances the mating success of sons); as expected the sex ratio is slightly less female biased than under local mate competition when both forces operate $(r=(n-1) / 2 n)$. When all males but no females disperse we have a model for local resource competition among females. Hamilton, quoted by Charnov (1982, pp. 74-75), obtained the equilibrium sex ratio $n /(2 n-1)$ for this model. Table $2 \mathrm{c}$ together with (5) shows that this is exact under male control of the sex ratio but only approximate under female control.

Acknowledgements I thank N. Barton, S.. A. Frank, A. Grafen and W. D. Hamilton for valuable comments.

\section{REFERENCES}

BULMER, M. G. AND TAYLOR, P. D. 1980. Dispersal and the sex ratio. Nature, $284,448-449$.
CHARnOV, E. L. 1982. The Theory of Sex Allocation. Princeton University Press.

FELleR, w. 1968. Introduction to Probability Theory and its Applications, Vol. 1, Wiley, New York.

FISHER, R. A. 1930. The Genetical Theory of Natural Selection. Oxford University Press.

FRANK, S. A. 1985. Hierarchical selection theory and sex ratios. II. On applying the theory, and a test with fig wasps. Evolution, in press.

GRAFEN, A. 1984. Natural selection, kin selection and group selection. In Behavioural Ecology (J. R. Krebs and N. B. Davies eds), pp. 62-84. Blackwell, Oxford.

HAMILTON, w. D. 1967. Extraordinary sex ratios. Science, 156, 477-488.

HAMILTON, W. D. 1979. Wingless and fighting males in fig wasps and other insects. In Reproductive Competition and Sexual Selection in Insects (M. S. Blum and N. A. Blum eds), pp. 167-220. Academic Press, New York.

HARVEY, P. H. 1985. Intrademic group selection and the sex ratio. In Behavioural Ecology (R. M. Sibley and R. H. Smith eds), pp. 59-73. Blackwell, Oxford.

MAYNARD SMITH, J. 1985. Appendix to Harvey (1985).

TAYLOR, P. D. 1981. Intra-sex and inter-sex sibling interactions as sex ratio determinants. Nature, 291, 64-66.

TAYLOR, P. D. AND BULMER, M. G. 1980. Local mate competition and the sex ratio. J. Theor. Biol., 86, 409-419.

VAN TIENDEREN, P. H. 1984. Eigenvalues and the search for evolutionary stable strategies in patchy model populations. Nieuw Arch. Wisk., 2, 25-42.

WERREN, J. H. 1983. Sex ratio evolution under local mate competition in a parasitic wasp. Evolution, 37, 116-124. 